\title{
Analog/RF Performance of Triple Material Gate Stack-Graded Channel Double Gate-Junctionless Strained-Silicon MOSFET with Fixed Charges
}

Subba Rao Suddapalli ( $\sim$ subbarao.s29@gmail.com )

National Institute of Technology Warangal https://orcid.org/0000-0002-3191-0731

Rani Deepika Balavendran Joseph

University of North Texas at Dallas

Vijaya Durga Chintala

National Institute of Technology Warangal

Gopi Krishna Saramekala

National Institute of Technology calicut

Srikar D

National Institute of Technology Warangal

Bheema Rao Nistala

National Institute of Technology Warangal

\section{Research Article}

Keywords: Fixed charges, Early voltage, Strained- silicon, Short channel effects, Transconductance.

Posted Date: September 21st, 2021

DOI: https://doi.org/10.21203/rs.3.rs-539499/v2

License: (1) This work is licensed under a Creative Commons Attribution 4.0 International License.

Read Full License 


\title{
Analog/RF performance of Triple Material Gate Stack-Graded Channel Double Gate-Junctionless strained-silicon MOSFET with fixed charges
}

\author{
Suddapalli Subba Rao*, Rani Deepika Balavendran Joseph**, Vijaya Durga

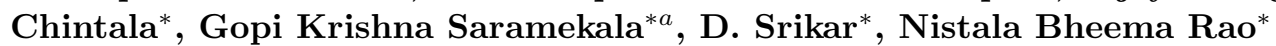

Received: 16 August 2021 / Accepted:

\begin{abstract}
In this paper, analog/radio frequency (RF) electrical characteristics of triple material gate stackgraded channel double gate-Junctionless (TMGS-GCDGJL) strained-Si (s-Si) MOSFET with fixed charge density is analyzed with the help of Sentaurus TCAD. By varying the various device parameters, the analog/RF performance of the proposed TMGS-GCDG-JL s-Si MOSFET is evaluated in terms of transconductance-generationfactor (TGF), early voltage, voltage gain, unity-powergain frequency $\left(f_{\max }\right)$, unity-current-gain frequency $\left(f_{t}\right)$, and gain-transconductance frequency product (GTFP). The results confirm that the proposed TMGS-GCDGJL s-Si MOSFET has superior analog/RF performance compared to gate stack-graded channel double gatejunctionless (GS-GCDG-JL) s-Si device. However, the proposed MOSFET has less transconductance and less output conductance when compared with the GS-GCDGJL s-Si device in above threshold region, and reverse trend follows in sub-threshold region.
\end{abstract}

Keywords Fixed charges · Early voltage - Strainedsilicon - Short channel effects - Transconductance.

\footnotetext{
* Dept. of ECE, NIT Warangal, Telangana, India, 506004. E-mail: subbarao.s29@gmail.com, vijayadurga20@gmail.com, srikard86@gmail.com, nbr.rao@gmail.com.

** Dept. of Electrical engineering, University of North Texas, Denton, TX, USA.

E-mail: ranideepika.balavendranjoseph@unt.edu.

*a Dept. of ECE, NIT Calicut, Kerala, India, 673601.

E-mail: gopikrishna@nitc.ac.in.
}

\section{Introduction}

Nano scaled strained-silicon (s-Si) MOSFETs are promising candidates for upcoming high-speed devices on account of high field velocity, enhanced mobility, and higher driving current [1]-[6]. With the aid of layer transfer process [7], the strain is developed in the silicon material. In this method, the biaxial-tensile strain is introduced in silicon material by growing the silicon material on the relaxed $S i_{(1-X)} G e_{(X)}$ material, which is developed on the Silicon-on-insulator (SOI) layer. When device operates in nano-scaled regime, fixed charges are created at Oxide/s-Si $\left(\mathrm{SiO}_{2} / \mathrm{s}-\mathrm{Si}\right)$ interface due to the lateral electric field in s-Si MOSFETs [8]-[9]. Thereby, the performance of MOSFET deteriorates in terms of threshold voltage and channel potential.

A few authors have presented the impact of fixed charges on the electrical characteristics of double gate (DG) junctionless device [10]-[12]. Also, they have confirmed that the fixed charges at the $\mathrm{SiO}_{2} / \mathrm{s}-\mathrm{Si}$ interface of MOSFET can cause change in the channel potential and threshold voltage. To suppress the hot carrier effects (HCEs), the triple metal gate (TMG) engineering is introduced in DG MOSFET [13]-[14]. In TMG structure, screen and control gates are used with three distinct work functions. Therefore, a step equivalent profile in s-Si channel potential and increase in average electric-field of s-Si channel are obtained. Hence, the performance of DG device is improved by employing the TMG structure. To further suppress HCEs, gate stack (GS) structure is also incorporated in DG MOSFET to achieve better device performance in terms of gate leakage current and drive current [15]-[16]. Furthermore, graded channel (GC) engineering is employed in DG junctionless device, thus resulting in improved analog/RF characteristics owing to diminished HCEs 
[17]-[18]. Therefore, better performance of the DG junctionless MOSFET is obtained by employing the GS with gate and channel engineering.

The analog/RF electrical characteristics of junctionless MOSFETs for low power applications was demonstrated in [19]. Moreover, enhancement in early voltage and intrinsic voltage gain are attained due to low electric field at drain end of oxide interface. In [20], the effect of laterally graded channel doping in double gate junctionless MOSFETs for analog/RF applications was presented. Besides, high cutoff frequency and high intrinsic voltage gain are obtained. In [21], the comparative analysis of DG junction less and GS DG junction less MOSFETs was presented. Also, the effect of doping on obtaining the optimum performance of the device was studied. In [22], the analytical modeling of leakage currents in dual material halo doped cylindrical gate junctionless MOSFET was illustrated. Till now, in literature, the analog/RF characteristics of triple material gate stack-graded channel DG-junctionless (TMGSGCDG-JL) s-Si device with the fixed charges has not been presented. By employing the GC and GS structure with gate engineering, better analog/RF performance of junctionless s-Si DG device is achieved.

This paper demonstrates the analysis of analog/RF characteristics of TMGS-GCDG-JL s-Si device with fixed charges. The analog/RF characteristics of TMGS-GCDG$\mathrm{JL}$ s-Si device is exhaustively analyzed by varying the strain $(m)$, fixed charge density $\left(N_{f}\right)$, and the thickness of high-k material $\left(t_{o x 2}\right)$. Moreover, the analog figure of merits of the proposed MOSFET are evaluated in terms of transconductance generation factor $\left(T G F=\left(\frac{g_{m}}{I_{d s}}\right)\right)$, early voltage $\left(\frac{I_{d s}}{g_{d s}}\right)$, and intrinsic voltage gain $\left(\frac{g_{m}}{g_{d}}\right)$. Besides, the RF figure of merits of the proposed device, unity current gain frequency $\left(f_{t}\right)$, unity power gain frequency $\left(f_{\max }\right)$ and gain transconductance frequency product $\left(G T F P=\left(\frac{g_{m}}{g_{d}}\right)\left(\frac{g_{m}}{I_{d}}\right) f_{t}\right)$, are evaluated exhaustively. The analog/RF characteristics of the proposed junctionless s-Si device are improved by increasing in the $t_{o x 2}, m$, and positive $N_{f}$ in the below threshold region, and vice-versa in above threshold voltage region. Also, better analog/RF electrical characteristics of the proposed TMGS-GCDGJL s-Si MOSFET are obtained when compared to GS graded channel DG junctionless (GS-GCDG-JL) s-Si MOSFET.

\section{Proposed MOSFET structure and TCAD setup}

The simulated cross sectional view of TMGS-GCDGJL s-Si device with fixed charges is demonstrated in
Fig. 1(a). The strained-silicon graded channel region is doped with three different uniform phosphorous doping concentrations (i.e., $N_{d 1}, N_{d 2}$, and $N_{d 3}$ ). Screen and control gates are used together to form bottom and top gates of TMGS-GCDG-JL s-Si device. The bottom and top gates of the GS-GCDG-JL s-Si MOSFET has a single gate layer whose work function is an average of $\phi_{m 1}, \phi_{m 2}$ and $\phi_{m 3}$. Because of HCEs in nano scaled proposed s-Si junctionless MOSFET, interface charges are created at s-Si/ $\mathrm{SiO}_{2}$ interface and are represented as damaged region of length $L_{d}$, as demonstrated in Fig. 1(a). The drain current of s-Si MOSFET is calibrated with the transfer characteristics of the strainedsilicon MOSFET using TCAD [23], as shown in Fig. 1(b). It is obvious from Fig. 1(b) that the TCAD simulation results of strained-silicon MOSFET are in good accordance with the experimental results shown in [23]. The various parameters and dimensions of the proposed TMGS-GCDG-JL s-Si MOSFET are used in the simulation are listed in Table 1.

Since the strain is developed into silicon channel, the energy-band diagram of the silicon material is affected because of the biaxial-tension. Thereby, energy band gap of silicon and effective mass of the carriers decrease, whereas electron affinity $\left(\chi_{S i}\right)$ increases. The changes in energy band gap, electron affinity, and effective masses of carrier in s-Si material are formulated as shown below $[24,25]$

$$
\begin{aligned}
& \left(\Delta E_{c}\right)_{s-S i}=0.57 X, \quad\left(\Delta E_{g}\right)_{s-S i}=0.4 X \\
& V_{T} \ln \left(\frac{N_{V, S i}}{N_{V, s-S i}}\right)=V_{T} \ln \left(\frac{m_{h, S i}^{*}}{m_{h, s-S i}^{*}}\right)^{\frac{3}{2}} \approx 0.075 X
\end{aligned}
$$

where $V_{T}$ denotes the thermal voltage, $N_{V, S i}$ and $N_{V, s-S i}$ represent density of states in the valence band, $m_{h, S i}^{*}$ and $m_{h, s-S i}^{*}$ denote the effective masses of hole in silicon and strained-silicon, respectively. Also, both flat band voltage and barrier potential of the source (drain) to channel decrease simultaneously [26].

$$
\begin{aligned}
& \Delta V_{f b}=-\frac{E_{c}}{q}+\frac{E_{g}}{q}-V_{T} \ln \left(\frac{N_{V, S i}}{N_{V, s-S i}}\right) \\
& \Delta V_{b i}=V_{T} \ln \left(\frac{N_{V, S i}}{N_{V, s-S i}}\right)-\frac{E_{g}}{q}
\end{aligned}
$$

where $\Delta V_{f b}$ and $\Delta V_{b i}$ are the changes in flat band voltage and built-in potential, respectively.

The proposed device with the fixed charges is simulated using the TCAD [27]. In the device simulation, to evaluate the analog/RF characteristics of the TMGSGCDG-JL s-Si deice with fixed charges, the following physical models are considered. The charge transport mechanism is estimated with the help of the drift diffusion model and the recombination of carriers are determined by using Auger and SRH recombination models. And also, mobility of carriers is estimated by us- 


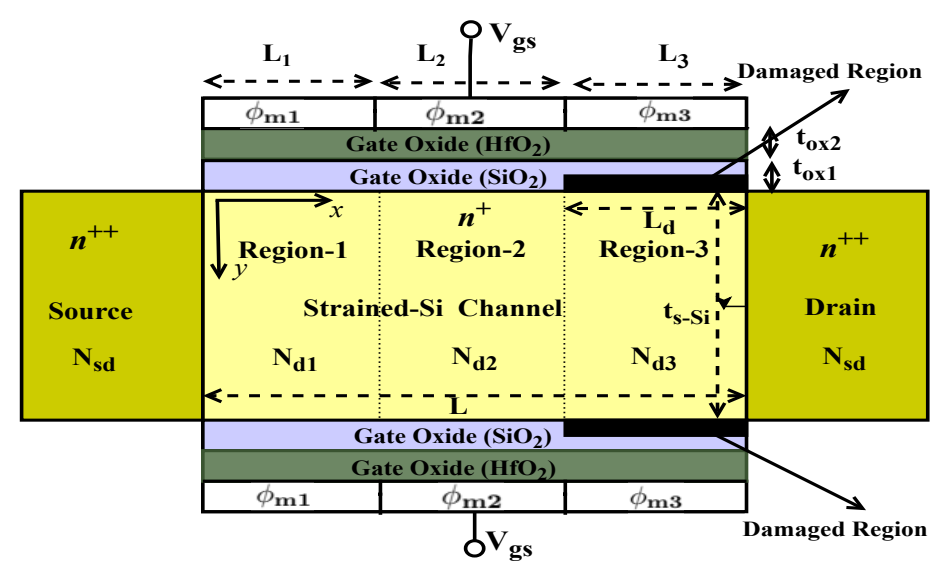

(a)

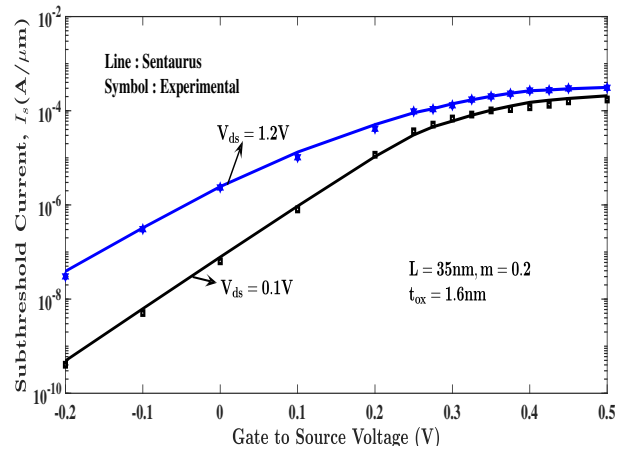

(b)

Fig. 1: Symmetrical TMGS-GCDG-JL s-Si MOSFET fixed charges a. Proposed junctionless MOSFET structure b. Comparison of strained-silicon MOSFET with experimental results of the [23].

Table 1: Parameters are employed in the simulation of TMGS-GCDG-JL s-Si MOSFET

\begin{tabular}{|l|l|l|l|}
\hline S. No. & Variable & Symbol & Values \\
\hline 1 & strained-silicon channel length & $L$ & $15 \mathrm{~nm}$ \\
\hline 2 & strained-silicon channel doping & $N_{d 1}, N_{d 2}, N_{d 3}$ & $10^{18}, 5 \times 10^{17}, 10^{17} \mathrm{~cm}^{-3}$ \\
\hline 3 & Source (Drain) doping & $N_{s d}$ & $10^{20} \mathrm{~cm}^{-3}$ \\
\hline 4 & strained-silicon thickness & $t_{s-S i}$ & $6 \mathrm{~nm}$ \\
\hline 5 & Oxide thicknesses & $t_{o x 1}, t_{o x 2}$ & $0.6,1 \mathrm{~nm}$ \\
\hline 6 & Work function of the control gate and screen gate & $\phi_{m 1}, \phi_{m 2}, \phi_{m 3}$ & $4.8,4.6,4.4 \mathrm{eV}$ \\
\hline 7 & $V_{g s}$ & $0-1 \mathrm{~V}$ \\
\hline 8 & Gate to source voltage & $V_{d s}$ & $0-1 \mathrm{~V}$ \\
\hline 9 & Drain to source voltage & $m$ & $0.1-0.3$ \\
\hline 10 & Ge mole fraction & $N_{f}$ & $-4 \times 10^{12}-4 \times 10^{12} \mathrm{~cm}^{-2}$ \\
\hline 11 & Fixed charges & $f_{0}$ & $0.1-1000 \mathrm{GHz}$ \\
\hline
\end{tabular}

ing Enormal mobility model and high field saturation model. Moreover, the energy band gap narrowing effects are considered by the OldSlotboom model and strainedsilicon characteristics are assessed by MoleFraction model. Furthermore, the effect of fixed charges at $\mathrm{SiO}_{2} / \mathrm{s}-\mathrm{Si}$ interface of the device are included with the help of Traps model and quantum mechanical effects are also assessed with the help of density gradient model [28].

\section{Result analysis}

This section demonstrates the analog and radio frequency performance of the TMGS-GCDG-JL s-Si MOSFET with fixed charges using the simulation results obtained from TCAD. Fig. 2 depicts the effects of $m$ on the $g_{m}$ and transfer characteristics of TMGS-GCDG-JL s-Si device. It is noticed from the Fig. 2 that the GSGCDG-JL s-Si device has enhanced transport characteristics and $g_{m}$ compared to proposed TMGS-GCDGJL s-Si device because of the lower threshold voltage of GS-GCDG-JL s-Si device. Moreover, increment in both ON current and $g_{m}$ of the TMGS-GCDG-JL s-Si device are observed in subthreshold region by increasing the $m$ because of decrease in the threshold voltage.

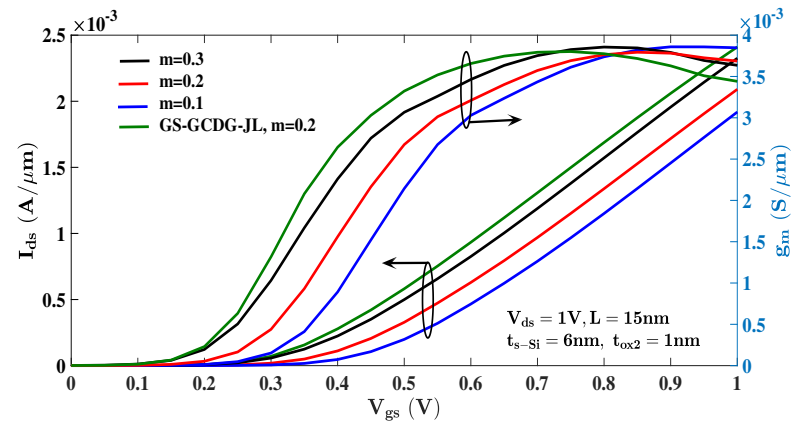

Fig. 2: Effect of strain in silicon channel on the transfer characteristics and $g_{m}$ of the TMGS-GCDG-JL s-Si device.

The variation of $t_{o x 2}$ on $g_{m}$ and transfer characteristics of TMGS-GCDG-JL s-Si MOSFET is shown in Fig. 3. When $t_{o x 2}$ decreases, increment in both $g_{m}$ and transfer characteristics of MOSFET are observed in the above threshold region because of the greater gate control over the s-Si channel than drain and reverse trend follows in weak inversion region. Besides, the gate stack that consists of $\mathrm{HfO}_{2}$ in the TMGS-GCDG-JL sSi MOSFET has better $g_{m}$ and transfer characteristics 


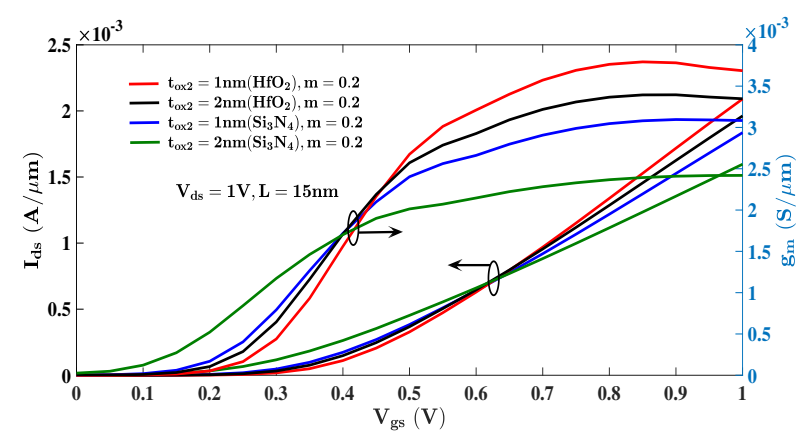

Fig. 3: Variation of $t_{o x 2}$ on the $g_{m}$ and transfer characteristics of the TMGS-GCDG-JL s-Si MOSFET.

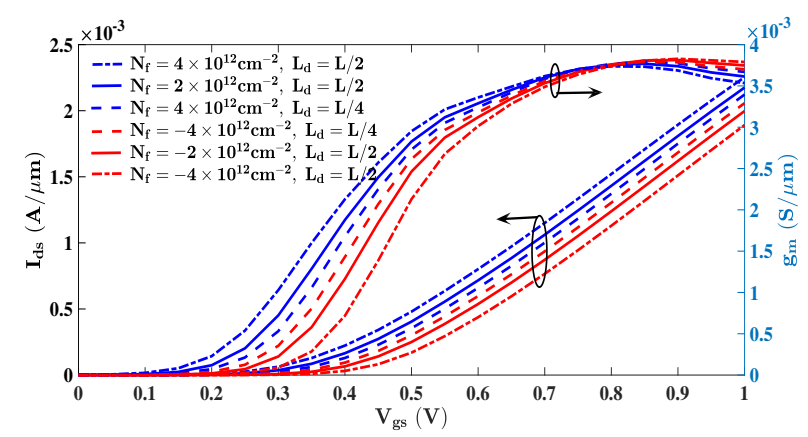

Fig. 4: Variation of $N_{f}$ on the transfer characteristics and $g_{m}$ of TMGS-GCDG-JL s-Si device.

than the gate stack that consists of $\mathrm{Si}_{3} \mathrm{~N}_{4}$ in the above threshold region and inversely in subthreshold region.

The variation of $N_{f}$ with $L_{d}$ on the transfer characteristics and $g_{m}$ of the proposed TMGS-GCDG-JL s-Si MOSFET is depicted in Fig. 4. The enhanced transfer characteristics of the TMGS-GCDG-JL s-Si device are attained by increasing positive $N_{f}$ since the threshold voltage of device decreases, and reverse trend follows for the negative $N_{f}$. Moreover, as positive/negative fixed charge density increases, the transconductance of TMGSGCDG-JL s-Si MOSFET increases in sub-threshold region and reverse trend follows in strong inversion region. Hence, the analog/RF characteristics of the proposed TMGS-GCDG-JL s-Si MOSFET are affected with respect to the fixed charges at $\mathrm{SiO}_{2} / \mathrm{s}-\mathrm{Si}$ interface.

Fig. 5 depicts the variation of $m$ on the output characteristics and $g_{d}$ of TMGS-GCDG-JL s-Si MOSFET. As strain increases, better drain characteristics and high $g_{d}$ of the TMGS-GCDG-JL s-Si MOSFET are attained because of decrease in the threshold voltage of proposed MOSFET. It is evident from Fig. 5 that the GS-GCDG-JL s-Si device has enhanced drain characteristics and higher $g_{d}$ compared to the proposed TMGS-GCDG-JL s-Si MOSFET. Therefore, the proposed TMGS-GCDG-JL s-Si MOSFET has lower effect of drain to source voltage on the channel compared to the GS-GCDG-JL s-Si MOSFET.

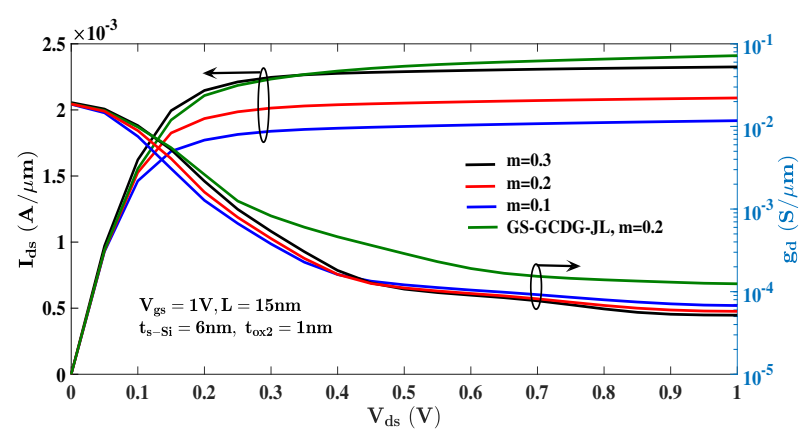

Fig. 5: Effect of $m$ on the drain characteristics and output conductance of TMGS-GCDG-JL s-Si device.

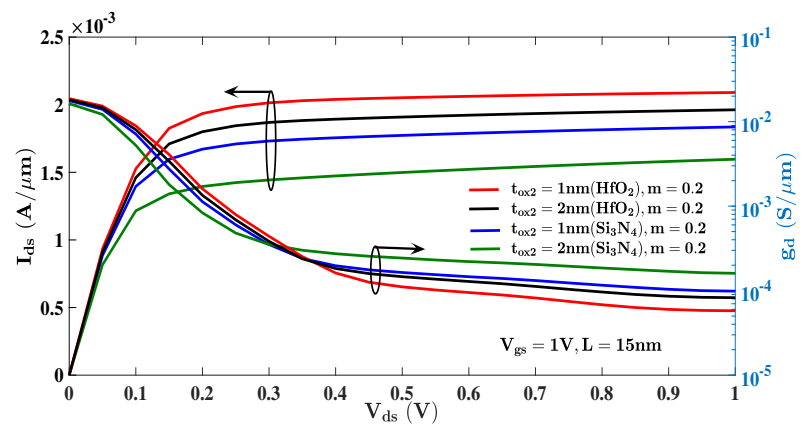

Fig. 6: Variation of $t_{o x 2}$ on the drain characteristics and $g_{d}$ of TMGS-GCDG-JL s-Si device.

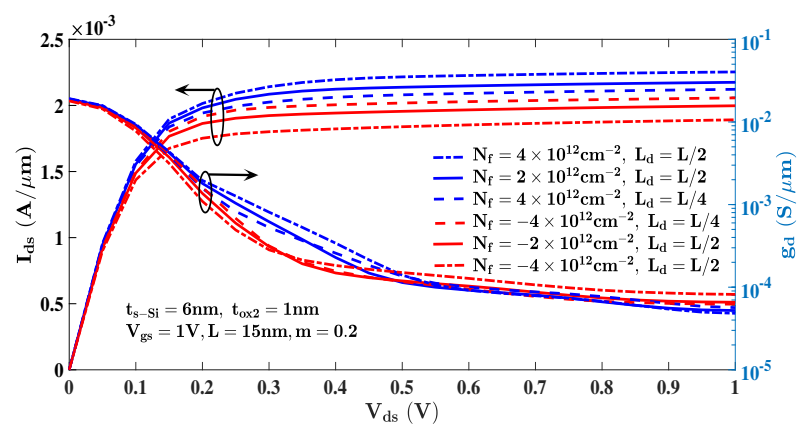

Fig. 7: Variation of $N_{f}$ with $L_{d}$ on output characteristics and $g_{d}$ of TMGS-GCDG-JL s-Si MOSFET.

The variation of $t_{o x 2}$ on output characteristics and $g_{d}$ of the TMGS-GCDG-JL s-Si MOSFET is illustrated in Fig. 6. As $t_{o x 2}$ decreases, $g_{d}$ decreases and the proposed MOSFET achieves better drain characteristics. Besides, the gate stack that consists of $\mathrm{HfO}_{2}$ in the TMGS-GCDG-JL s-Si MOSFET has improved drive current and less $g_{d}$ than the gate stack that consists of $\mathrm{Si}_{3} \mathrm{~N}_{4}$. Therefore, $\mathrm{HfO}_{2} / \mathrm{SiO}_{2}$ gate stack of proposed TMGS-GCDG-JL s-Si has better electrical characteristics and less impact of $V_{d s}$ on the channel when compared to the $\mathrm{Si}_{3} \mathrm{~N}_{4} / \mathrm{SiO}_{2}$ gate stack, as shown in Fig. 6 .

Fig. 7 illustrates the effect of $N_{f}$ with $L_{d}$ on output characteristics and $g_{d}$ of the TMGS-GCDG-JL s- 


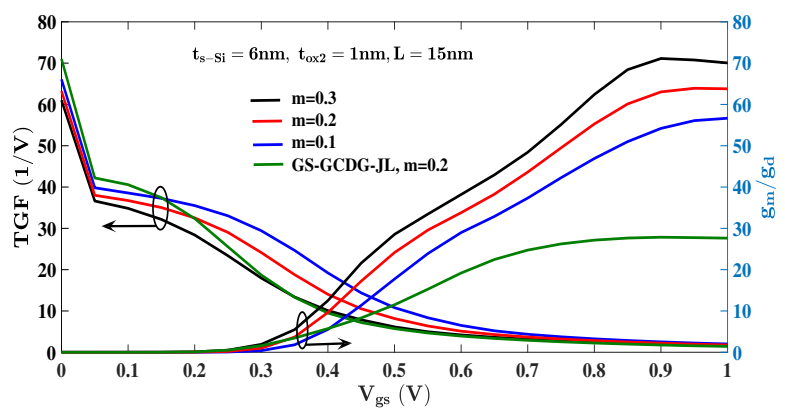

Fig. 8: Effect of $m$ on the intrinsic voltage gain and TGF of TMGS-GCDG-JL s-Si device.

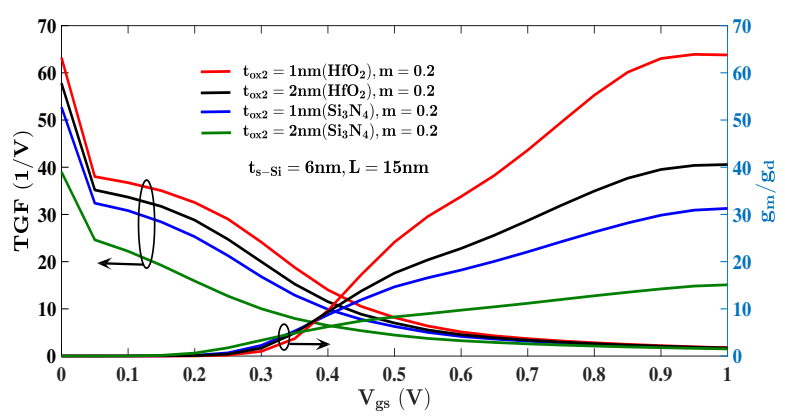

Fig. 9: Variation of $t_{o x 2}$ on the intrinsic voltage gain and TGF of TMGS-GCDG-JL s-Si device.

Si MOSFET. Increase in output characteristics is observed by increasing the positive $N_{f}$ owing to reduced threshold voltage of device, and vice-versa when $N_{f}$ is negative. Moreover, as positive $N_{f}$ increases, $g_{d}$ of proposed device decreases, and vice-versa when $N_{f}$ is negative. Therefore, the proposed TMGS-GCDG-JL s-Si MOSFET with positive $N_{f}$ has lower impact of $V_{d s}$ on the channel compared to the GS-GCDG-JL s-Si MOSFET with negative $N_{f}$.

The variation of strain on TGF and intrinsic gain of TMGS-GCDG-JL s-Si is depicted in Fig. 8. As strain increases in the TMGS-GCDG-JL s-Si MOSFET, TGF decreases and intrinsic voltage gain increases owing to increasing the values of transconductance and drain current of the proposed device, as depicts in Fig. 2. When compared to GS-GCDG-JL s-Si device, TMGSGCDG-JL s-Si device has better TGF and higher $\frac{g_{m}}{g_{d}}$ due to high $g_{m}$ and low $g_{d}$ of the TMGS-GCDG-JL sSi device, as illustrated in Fig. 2 and Fig. 5. Thereby, the proposed device has higher analog voltage gain and superior power efficiency due to TMG structure when compared to GS-GCDG-JL s-Si device.

Fig. 9 plots the effect of $t_{o x 2}$ on the voltage gain and TGF of the TMGS-GCDG-JL s-Si MOSFET. The power conversion efficiency and voltage gain of proposed MOSFET decrease because of the less gate control over the strained-Si channel than drain terminal as

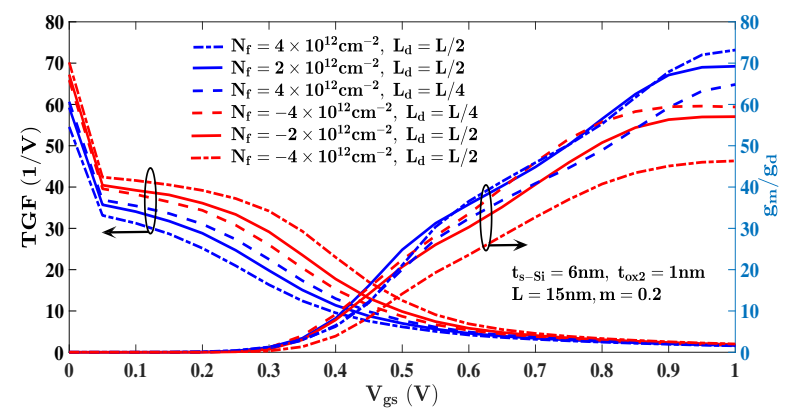

Fig. 10: Variation of $N_{f}$ on the intrinsic voltage gain and TGF of the TMGS-GCDG-JL s-Si device.

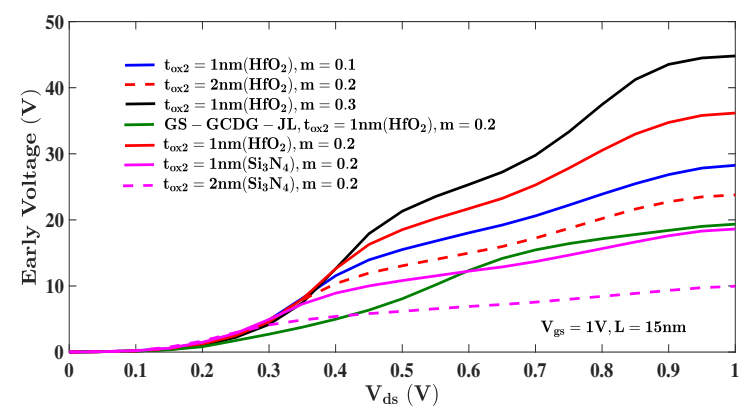

Fig. 11: Variations of $t_{o x 2}$ and $m$ on the early voltage of TMGS-GCDG-JL s-Si device

$t_{o x 2}$ increases. Moreover, gate stack with $\mathrm{HfO}_{2}$ has better TGF and higher voltage gain than the gate stack with $\mathrm{Si}_{3} \mathrm{~N}_{4}$ of the TMGS-GCDG-JL s-Si MOSFET due to the higher $g_{m}$ and lower $g_{d}$ of the TMGS-GCDG-JL s-Si MOSFET, as illustrated in Fig. 3 and Fig. 6. Therefore, the proposed MOSFET with $\mathrm{Si}_{3} \mathrm{~N}_{4}$ has higher analog intrinsic gain and low power consumption are obtained.

The effect of $N_{f}$ on the intrinsic voltage gain and TGF of the TMGS-GCDG-JL s-Si device is shown in Fig. 10. As positive $N_{f}$ increases, TGF decreases and intrinsic voltage gain increases owing to the decrease in threshold voltage of TMGS-GCDG-JL s-Si MOSFET, and vice-versa for negative fixed charge density. Hence, the TMGS-GCDG-JL s-Si MOSFET with negative fixed charge density can be operated at lower bias values, and vice-versa for positive $N_{f}$. Moreover, the proposed MOSFET with positive $N_{f}$ has higher analog intrinsic gain is attained.

Fig. 11 shows the variations of $m$ and $t_{o x 2}$ on the early voltage of TMGS-GCDG-JL s-Si device. The early voltage of the TMGS-GCDG-JL s-Si MOSFET increases as $m$ increases and $t_{o x 2}$ decreases since the $g_{d}$ of the device decreases, as illustrated in Fig. 5 and Fig. 6 (low early voltage denotes the high channel length modulation effect in the MOSFET). Besides, gate stack with $\mathrm{HfO}_{2}$ has greater early voltage than the gate stack with 


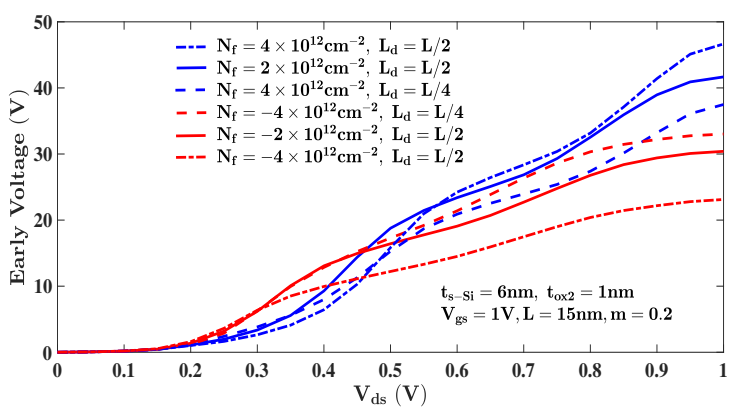

Fig. 12: Variation of the $N_{f}$ on the early voltage of TMGS-GCDG-JL s-Si device.

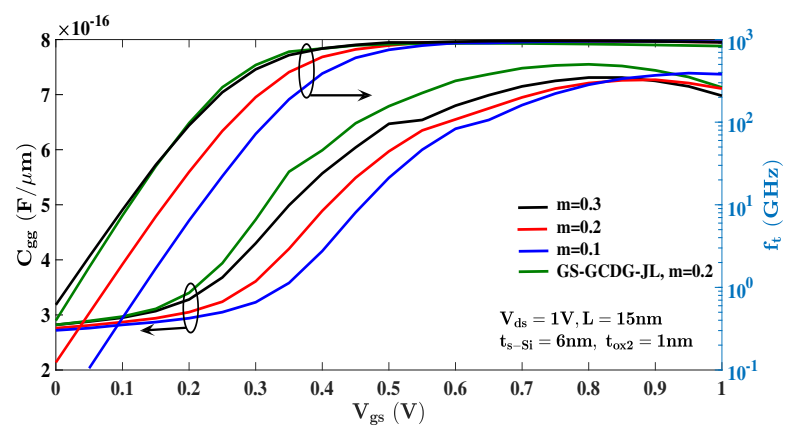

Fig. 13: Effect of the strain on the gate capacitance and $f_{t}$ of TMGS-GCDG-JL s-Si device.

$\mathrm{Si}_{3} \mathrm{~N}_{4}$ of the TMGS-GCDG-JL s-Si MOSFET since the output conductance for gate stack with $\mathrm{HfO}_{2}$ is less, as depicts in Fig. 6. Moreover, the proposed TMGSGCDG-JL s-Si MOSFET has higher early voltage than the GS-GCDG-JL s-Si MOSFET due to the less output conductance of the proposed device, as depicts in Fig. 5. As a result, the effect of channel length modulation in proposed TMGS-GCDG-JL s-Si MOSFET is less compared to the GS-GCDG-JL s-Si MOSFET.

Fig. 12 demonstrates the effect of $N_{f}$ on early voltage of the TMGS-GCDG-JL s-Si MOSFET. The early voltage of the proposed device decreases/increases by increasing the negative/positive fixed charge density due to a increment/decrement in the output conductance with respect to negative/positive $N_{f}$, as demonstrated in Fig. 7. Hence, effect of channel length modulation in the device increases/decreases due to negative/positive fixed $N_{f}$ at s-Si/oxide interface.

Fig. 13 demonstrates the effect of $m$ on $f_{t}$ and total gate capacitance of the TMGS-GCDG-JL s-Si MOSFET. As $m$ decreases, the $f_{t}$ and gate capacitance of the TMGS-GCDG-JL s-Si MOSFET decrease owing to an increment in the flat-band voltage of TMGS-GCDG$\mathrm{JL} \mathrm{s-Si} \mathrm{device} \mathrm{and} \mathrm{reduction} \mathrm{of} \mathrm{inversion} \mathrm{carriers} \mathrm{in}$ strained-silicon channel. Moreover, when compared to proposed TMGS-GCDG-JL s-Si device, the gate capacitance and $f_{t}$ of the GS-GCDG-JL s-Si MOSFET are

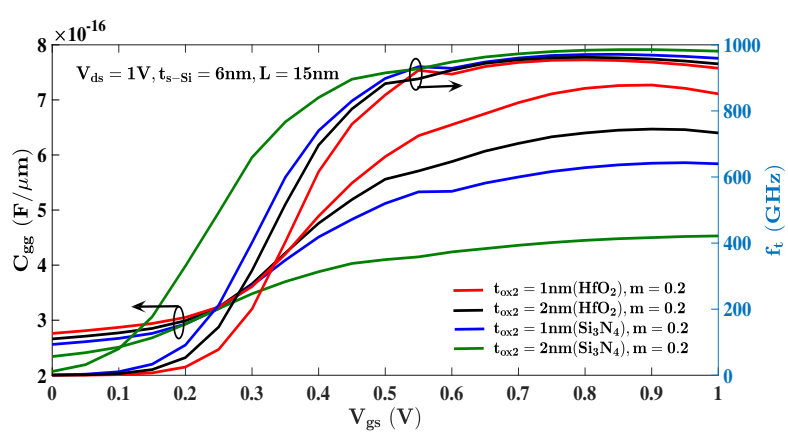

Fig. 14: Variation of $t_{o x 2}$ on gate capacitance and $f_{t}$ of TMGS-GCDG-JL s-Si MOSFET.

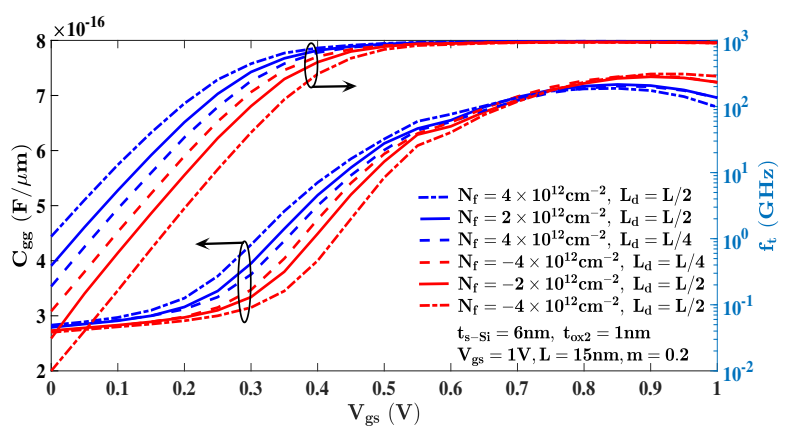

Fig. 15: Variation of $N_{f}$ on the $f_{t}$ and gate capacitance of TMGS-GCDG-JL s-Si device.

higher due to the higher $g_{m}$ of the GS-GCDG-JL s-Si device, as shown in Fig. 2.

The variation of $t_{o x 2}$ on the $f_{t}$ and gate capacitance of the TMGS-GCDG-JL s-Si device is depicted in Fig. 14. As $t_{o x 2}$ decreases, higher $C_{g g}$ and lower $f_{t}$ of the TMGS-GCDG-JL s-Si MOSFET are obtained due to the increase in inversion carriers in the channel. Besides, the gate stack with $\mathrm{HfO}_{2}$ has higher gate capacitance than the gate stack with $\mathrm{Si}_{3} \mathrm{~N}_{4}$ of the TMGS-GCDG-JL s-Si MOSFET due to the higher permittivity of $\mathrm{HfO}_{2}$. However, the gate stack with $\mathrm{HfO}_{2}$ has less $f_{t}$ than the gate stack with $\mathrm{Si}_{3} \mathrm{~N}_{4}$ of the TMGS-GCDG-JL s-Si MOSFET is noticed.

Fig. 15 shows the effect of fixed charged density on the gate capacitance and $f_{t}$ of the TMGS-GCDG-JL sSi device. As negative $N_{f}$ increases, gate capacitance of the TMGS-GCDG-JL s-Si MOSFET decreases due to mitigate of inversion charges in channel of MOSFET, and reverse trend follows for positive $N_{f}$. Moreover, as negative/positive $N_{f}$ increases, $f_{t}$ of the TMGSGCDG-JL s-Si device decreases/increases because of the decrement/increment in $g_{m}$ of TMGS-GCDG-JL s-Si MOSFET with negative/positive $N_{f}$, as demonstrated in Fig. 4.

Fig. 16 shows the variation of $t_{o x 2}$ on the voltage gain of the TMGS-GCDG-JL s-Si MOSFET with the operating frequency. As seen in Fig. 16, as $t_{o x 2}$ de- 


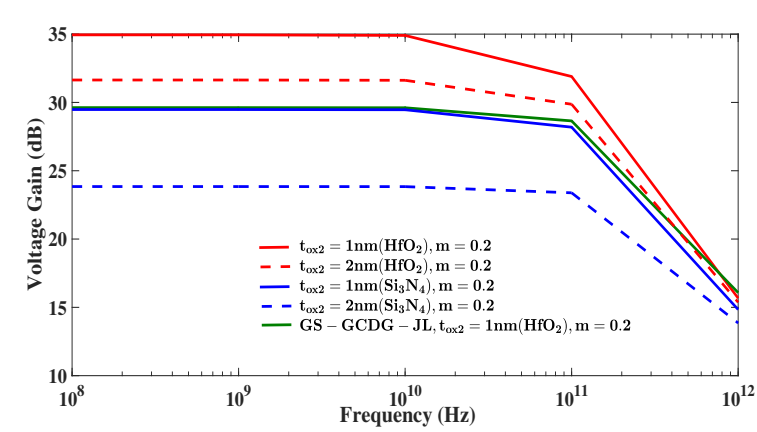

Fig. 16: variation of the $t_{o x 2}$ on the voltage gain of the TMGS-GCDG-JL s-Si device along operating frequency.

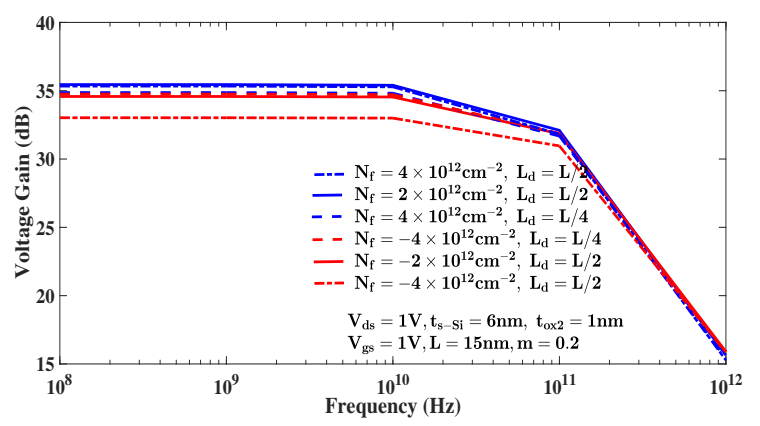

Fig. 17: Variation of $N_{f}$ on the voltage gain of TMGSGCDG-JL s-Si device along operating frequency.

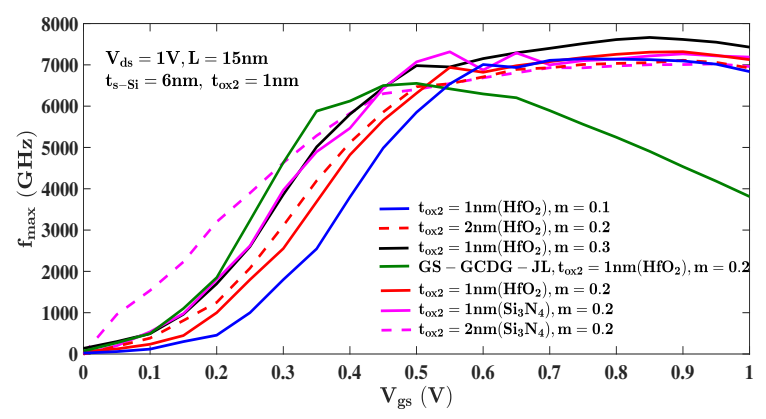

Fig. 18: Variations of strain and $t_{o x 2}$ on $f_{\max }$ of the TMGS-GCDG-JL s-Si MOSFET.

creases, voltage gain of TMGS-GCDG-JL s-Si device increases because of decrement in $g_{d}$ and increment in $g_{m}$, as shown in Fig. 2 and Fig. 5. Moreover, gate stack with $\mathrm{HfO}_{2}$ has higher voltage gain than the gate stack with $\mathrm{Si}_{3} \mathrm{~N}_{4}$ of the TMGS-GCDG-JL s-Si MOSFET. However, as operating frequency increases, voltage gain of proposed MOSFET decreases owing to the more parasitic capacitive effects. Also, the proposed TMGS-GCDG-JL s-Si MOSFET has greater voltage gain than GS-GCDG-JL s-Si device because of the TMG structure in the proposed device. Fig. 17 depicts the effect of fixed charge density on the voltage gain of the TMGS-GCDG-JL s-Si MOSFET. As positive/negative

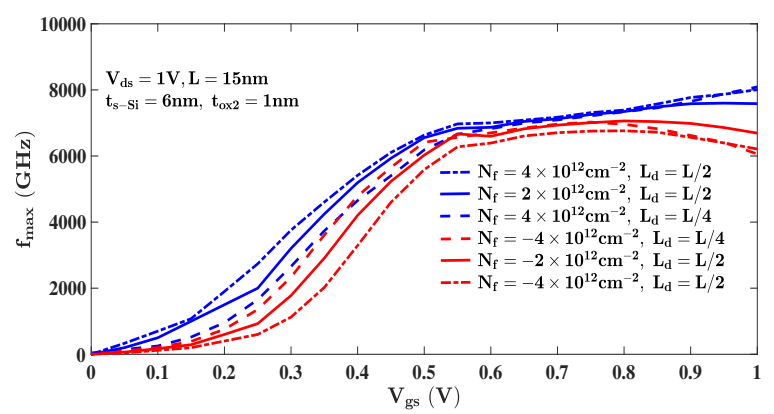

Fig. 19: Variation of $N_{f}$ on $f_{\max }$ of the TMGS-GCDGJL s-Si MOSFET.

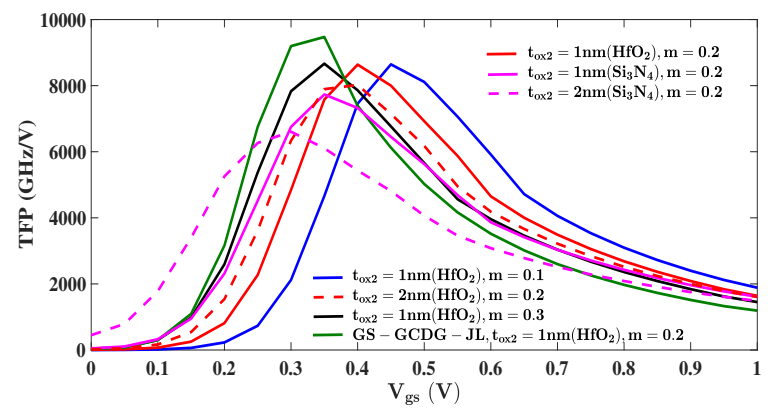

Fig. 20: Variations of the $m$ and $t_{o x 2}$ on the TFP of TMGS-GCDG-JL s-Si device.

$N_{f}$ increases, voltage gain of TMGS-GCDG-JL s-Si device increases/decreases owing to increment in $g_{m}$ and decrement in $g_{d}$, as shown in Fig. 4 and Fig. 7.

The variations of $m$ and $t_{o x 2}$ on $f_{\max }$ of the TMGSGCDG-JL s-Si MOSFET are shown in Fig. 18. Since $m$ increases and $t_{o x 2}$ decreases, $f_{\max }$ of the TMGSGCDG-JL s-Si MOSFET increases because of higher $g_{m}$ of proposed MOSFET, as shown in Fig. 2 and Fig.3. Besides, higher $f_{\text {max }}$ is attained for the $\mathrm{HfO}_{2} / \mathrm{SiO}_{2}$ gate stack in comparison with the $\mathrm{Si}_{3} \mathrm{~N}_{4} / \mathrm{SiO}_{2}$ gate stack of the TMGS-GCDG-JL s-Si device since higher $g_{m}$ of proposed MOSFET, as shown in Fig. 4. Moreover, the TMGS-GCDG-JL s-Si MOSFET has higher $f_{\max }$ in comparison with the GS-GCDG-JL s-Si device. The effect of $N_{f}$ on $f_{\max }$ of the TMGS-GCDG-JL s-Si device is illustrated in Fig. 19. It is evident from Fig. 19 that the increment/decrement in $f_{\max }$ of the TMGS-GCDGJL s-Si MOSFET is obtained as positive/negative $N_{f}$ increases with damaged length since higher/lower $g_{m}$ of proposed MOSFET with positive/negative $N_{f}$ at sSi/oxide interface, as shown in Fig. 4.

The variations of $m$ and $t_{o x 2}$ on TFP of s-Si TMGSGCDG-JL s-Si device are shown in Fig. 20. As seen from Fig. 20, it is evident that the TFP of the proposed MOSFET is improved by decreasing the values of $m$ and $t_{o x 2}$ in the above threshold voltage region and reverse trend follows in weak inversion region. Be- 


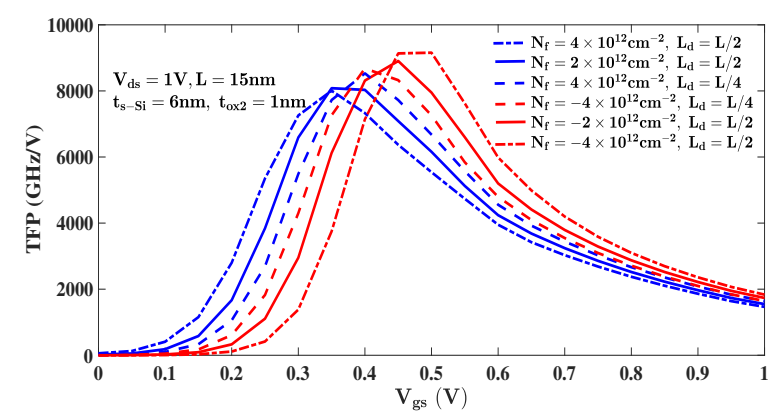

Fig. 21: Variation of the $N_{f}$ on TFP of TMGS-GCDGJL s-Si MOSFET.

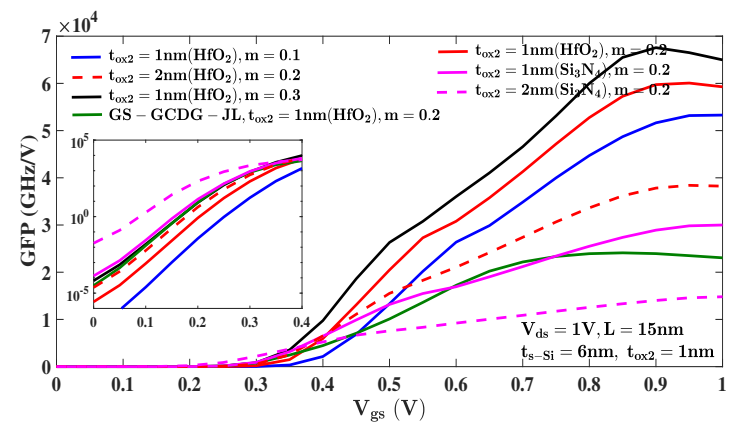

Fig. 22: Variations of $m$ and $t_{o x 2}$ on the GFP of the TMGS-GCDG-JL s-Si MOSFET.

sides, better TFP is obtained for the $\mathrm{HfO}_{2} / \mathrm{SiO}_{2}$ gate stack in comparison with the $\mathrm{Si}_{3} \mathrm{~N}_{4} / \mathrm{SiO}_{2}$ gate stack of the TMGS-GCDG-JL s-Si MOSFET. Also, TFP of the proposed TMGS-GCDG-JL s-Si MOSFET is more than the GS-GCDG-JL s-Si MOSFET in above subthreshold region, and reverse trend follows in weak inversion region. The effect of fixed charge density on the TFP of the TMGS-GCDG-JL s-Si MOSFET is shown in Fig. 21. TFP of the TMGS-GCDG-JL s-Si device decreases/increases as positive/negative $N_{f}$ increases in the strong inversion region, and reverse trend follows in weak inversion region.

Fig. 22 The variations of $t_{o x 2}$ and $m$ on the GFP of TMGS-GCDG-JL s-Si device are depicted in Fig. 22. GFP of the TMGS-GCDG-JL s-Si MOSFET decreases as strain and $t_{o x 2}$ increase in the above threshold region, and reverse trend follows in weak inversion region. Moreover, enhanced GFP is obtained for the $\mathrm{HfO}_{2} / \mathrm{SiO}_{2}$ gate stack in comparison with the $\mathrm{Si}_{3} \mathrm{~N}_{4} / \mathrm{SiO}_{2}$ gate stack of the TMGS-GCDG-JL s-Si MOSFET. Besides, the proposed TMGS-GCDG-JL s-Si MOSFET achieves higher GFP than the GS-GCDG-JL s-Si device in the above threshold voltage region, and reverse trend follows in weak inversion region. The variation of fixed charge density on GFP of the TMGS-GCDG-JL $\mathrm{s}-\mathrm{Si}$ device is depicted in Fig. 23. As negative (positive)

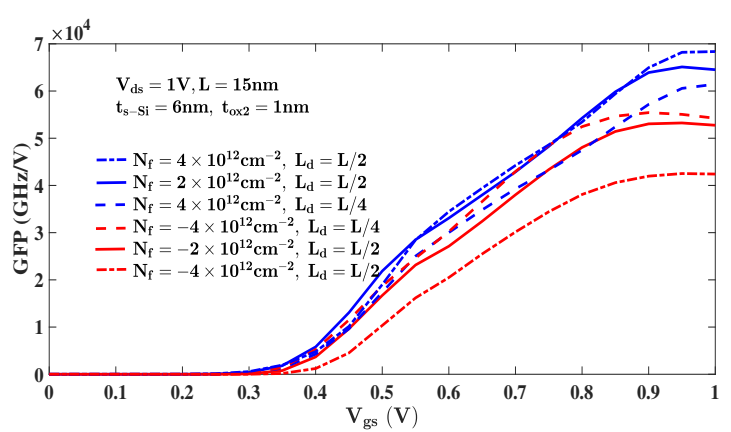

Fig. 23: Variation of the $N_{f}$ the TFP of TMGS-GCDGJL s-Si MOSFET.

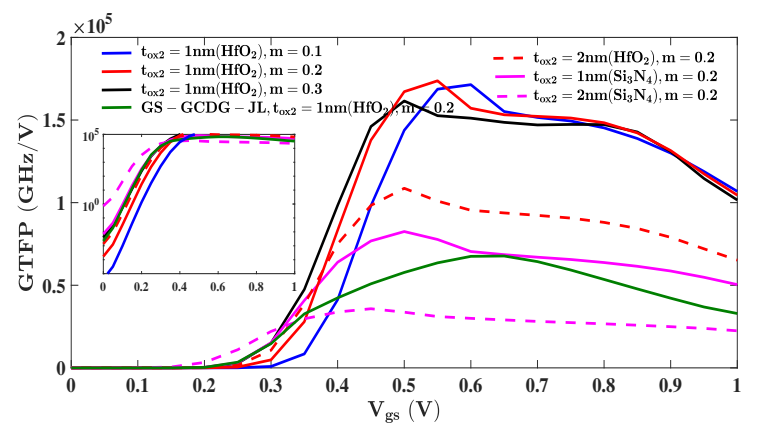

Fig. 24: Variations of $m$ and $t_{o x 2}$ on the GTFP of the TMGS-GCDG-JL s-Si MOSFET.

interface charge density increases, GFP of the TMGSGCDG-JL s-Si MOSFET decreases (increases).

The variations of $m$ and $t_{o x 2}$ on GTFP of the TMGSGCDG-JL s-Si MOSFET are depicted in Fig. 24. The GTFP of the TMGS-GCDG-JL s-Si MOSFET is increased by decreasing strain and $t_{o x 2}$ in above threshold region, and reverse trend follows in weak inversion region. Besides, $\mathrm{HfO}_{2} / \mathrm{SiO}_{2}$ gate-stack has greater GTFP when compared to $\mathrm{Si}_{3} \mathrm{~N}_{4} / \mathrm{SiO}_{2}$ gate stack of the TMGSGCDG-JL s-Si MOSFET. Moreover, it is obvious from Fig. 24 that the proposed device has improved overall figure of merit compared to the the GS-GCDG-JL s-Si device in the above threshold region.

Fig. 25 plots the variation of $N_{f}$ on GTFP of TMGSGCDG-JL s-Si device. For a given $L_{d}$, when $4 \times 10^{12} \leq$ $N_{f} \leq-2 \times 10^{12}$, it is observed that GTFP of TMGSGCDG-JL s-Si MOSFET increases/decreases as positive/negative interface charge density increases in the sub-threshold region and reverse trend follows in above threshold region. Besides, GTFP of the TMGS-GCDGJL s-Si device reduces because of DIBL effect when $N_{f}>-2 \times 10^{12}$. Hence, the overall analog/RF performance of the proposed the TMGS-GCDG-JL s-Si MOSFET varies with respect to fixed charges.

The performance analysis of proposed TMGS-GCDGJL s-Si MOSFET is compared with existing works in the previous works, as demonstrated in Table 2. The 
Table 2: The performance analysis of TMGS-GCDG-JL s-Si MOSFET with literature.

\begin{tabular}{|l|l|l|l|l|l|l|l|}
\hline Device & $\mathbf{g}_{m}, \mathbf{S} / \mu \mathbf{m}$ & $\left(\frac{I_{d}}{g_{d}}\right), \mathbf{V}$ & $\left(\frac{g_{m}}{I_{d}}\right), \mathbf{V}^{-1}$ & $\frac{g_{m}}{g_{d}}$ & $\mathbf{C}_{g g}, \mathbf{f F} / \mu \mathbf{m}$ & $\mathbf{f}_{t}, \mathbf{G H z}$ & $\mathbf{G T F P},(\mathbf{T H z} / \mathbf{V})$ \\
\hline GS-GCDG-JL s-Si & 3.8 & 19.35 & 71.01 & 27.85 & 0.755 & 900.2 & 67.71 \\
\hline TMGS-GCDG-JL s-Si & 3.79 & 36.17 & 63.23 & 63.91 & 0.727 & 954.6 & 173.7 \\
\hline High-k DG-JL [14] & 2.1 & 50.1 & 40.1 & 60.1 & 1.5 & 250 & - \\
\hline DG-JL [19] & 0.26 & 1.28 & 26.7 & 33.88 & 0.4 & 139 & - \\
\hline GC DG-JL [20] & 2.0 & 14.3 & 11.5 & 43.0 & 0.6 & 580 & - \\
\hline s-Si GC-DMDG [29] & 3.9 & 11.2 & 33.26 & 22.59 & 0.924 & 787 & 54.13 \\
\hline
\end{tabular}

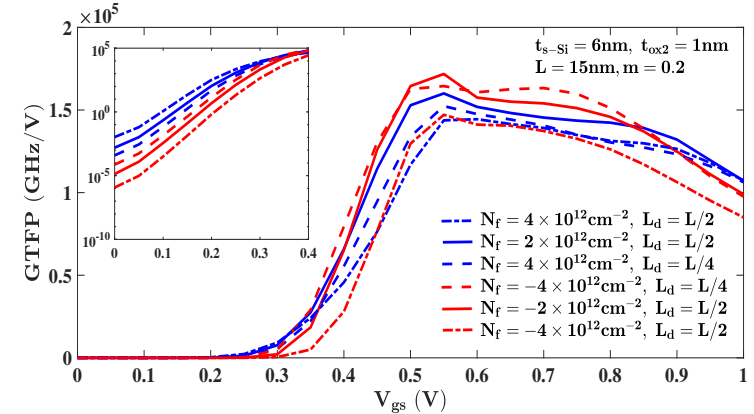

Fig. 25: Variation of $N_{f}$ on the GTFP of the TMGSGCDG-JL s-Si device.

voltage gain, $f_{t}$, and GTFP of proposed TMGS-GCDGJL s-Si MOSFET $\left(\mathrm{m}=0.2\right.$ and $\left.\mathrm{t}_{o x 2}=1 \mathrm{~nm}\right)$ are better than when compared to the GS-GCDG-JL s-Si MOSFET $\left(\mathrm{m}=0.2\right.$ and $\left.\mathrm{t}_{o x 2}=1 \mathrm{~nm}\right)$, high-k spacer DG junctionless MOSFET [14], DG junctionless MOSFET with channel length $20 \mathrm{~nm}$ [19], GC DG junctionless device with channel length $30 \mathrm{~nm}[20]$ and strained-Si GCDMDG MOSFET with channel length $20 \mathrm{~nm}$ [29]. However, the proposed TMGS-GCDG-JL s-Si MOSFET has lower early voltage than high-k spacer DG junctionless MOSFET [14]. Moreover, the proposed TMGS-GCDGJL s-Si MOSFET has lower $g_{m}$ than strained-Si GCDMDG MOSFET with channel length $20 \mathrm{~nm}$ [29]. Therefore, the proposed MOSFET using the TMG with GC engineering and gate stack techniques achieves improved overall analog/RF performance.

\section{Conclusion}

The analog/radio-frequency performance of the proposed TMGS-GCDG-JL s-Si MOSFET with fixed charge density at silicon dioxide interface has been estimated using TCAD tool. The proposed TMGS-GCDG-JL s-Si MOSFET has better overall analog/RF figure of merit compared to GS-GCDG-JL s-Si device in the above threshold region. Moreover, the analog/radio frequency figure of merits of TMGS-GCDG-JL s-Si device are improved by employing the TMG structure with the GC engineering and gate stack technique. An exhaustive analysis has been done to investigate the various
analog/RF characteristics by varying MOSFET parameters of the TMGS-GCDG-JL s-Si MOSFET. Increments in TFP and GTFP of proposed TMGS-GCDGJL s-Si MOSFET have been obtained by increasing values of strain, positive fixed charge density, and $t_{o x 2}$ in the weak inversion region, and vice-versa in the above threshold voltage region. Therefore, the proposed TMGSGCDG-JL s-Si MOSFET has better analog/radio-frequency figure of merits in above the threshold voltage region.

Acknowledgments: The authors gratefully acknowledge the simulation facilities provided by Analog IC lab, NIT Warangal.

Author Contributions: All the authors contributed to study conception and conceptualization. Material setup and TCAD simulation are performed by Suddapalli Subba Rao. Formal analysis and investigation of the simulated results were done by Rani Deepika Balavendran Joseph. The first draft of the manuscript was written by D. Srikar and Vijaya Durga Chintala and edited by Suddapalli Subba Rao and Gopi Krishna Saramekala. Finally, the complete work was carried under the supervision of Nistala Bheema Rao.

Funding: The authors declare that Suddapalli Subba Rao has received research support from National Institute of Technology Warangal. Further, authors have no other relevant funding or financial support to disclose in relevance to the work shown in this paper.

Data availability: The data that support the findings of this study are available from the corresponding author, upon reasonable request.

\section{Compliance with Ethical Standards}

Consent for Publication: All the authors declare their consent to transfer the publication rights to the journal in which this manuscript is submitted.

Consent to Participate: Not Applicable 
Conflicts of interest: The authors declare that there is no conflict of interests.

\section{References}

1. S. E. Thompson, M. Armstrong, C. Auth, S. Cea, R. Chau, G. Glass, T. Hoffman, J. Klaus, Z. Ma, B. Mcintyre, A. Murthy, B. Obradovic, L. Shifren, S. Sivakumar, S. Tyagi, T. Ghani, K. Mistry, M. Bohr, and Y. El-Mansy, "A logic nanotechnology featuring strained-silicon," IEEE Electron Device Letters, vol. 25, no. 4, pp. 191-193, April 2004.

2. M. Jurczak, T. Skotnicki, G. Ricci, Y. Campidelli, C. Hernandez, and D. Bensahel, "Study on Enhanced performance in NMOSFETs on Strained Silicon," in 29th European Solid-State Device Research Conference, vol. 1, Sep. 1999, pp. 304-307.

3. T. Sanuki, A. Oishi, Y. Morimasa, S. Aota, T. Kinoshita, R. Hasumi, Y. Takegawa, K. Isobe, H. Yoshimura, M. Iwai, K. Sunouchi, and T. Noguchi, "Scalability of strained silicon CMOSFET and high drive current enhancement in the $40 \mathrm{~nm}$ gate length technology," in IEEE International Electron Devices Meeting 2003, Dec 2003, pp. 3.5.1-3.5.4.

4. S. Keith, F. M. Bufler, and B. Meinerzhagen, "Full Band Monte-Carlo Device Simulation of an 0.1 um N-Channel MOSFET in Strained Silicon Material," in 27th European Solid-State Device Research Conference, Sep. 1997, pp. 200-203.

5. C. Nguyen, A. Pham, C. Jungemann, and B. Meinerzhagen, "Study of charge carrier quantization in strained SinMOSFETs," Materials Science in Semiconductor Processing, vol. 8, no. 1, pp. $363-366,2005$.

6. C. H. Ko, C. H. Ge, C. C. Huang, C. Y. Fu, C. P. Hsu, C. H. Chen, C. H. Chang, J. C. Lu, Y. C. Yeo, W. C. Lee, and M. H. Chi, "A novel process-induced strained silicon (PSS) CMOS technology for high-performance applications," in IEEE VLSI-TSA International Symposium on VLSI Technology, 2005. (VLSI-TSA-Tech)., April 2005, pp. 25-26.

7. T. Langdo, M. T. Currie, A. Lochtefeld, R. Hammond, J. Carlin, M. Erdtmann, G. Braithwaite, V. K. Yang, C. Vineis, H. Badawi, and M. Bulsara, "SiGe-free strained Si on insulator by wafer bonding and layer transfer," $A p$ plied Physics Letters, vol. 82, pp. 4256-4258, 062003.

8. S. R. Suddapalli and B. R. Nistala, "Analytical modeling of subthreshold current and swing of strained-Si graded channel dual material double gate MOSFET with interface charges and analysis of circuit performance," International Journal of Numerical Modelling: Electronic Networks, Devices and Fields, 2020.

9. R. Shankar, G. Kaushal, S. Maheshwaram, S. Dasgupta, and S. K. Manhas, "A Degradation Model of Double Gate and Gate-All-Around MOSFETs With Interface Trapped Charges Including Effects of Channel Mobile Charge Carriers," IEEE Transactions on Device and Materials Reliability, vol. 14, no. 2, pp. 689-697, 2014.

10. Yograj Singh Duksh, Balraj Singh, Deepti Gola, Pramod Kumar Tiwari and Satyabrata Jit, "Subthreshold Modeling of Graded Channel Double Gate Junctionless FETs," Silicon, vol. 13, pp. 1231-1238, 2021.

11. Md. Mahfuzul Haque, Md. Humaun Kabir and Md. Mohsinur Rahman Adnan, "Analytical modelling and verification of potential profile of DG JLFET with and without stack oxide," International Journal of Electronics, pp. 1-22, 2020 .
12. N. Vadthiya and K. A. Girdhardas, "Surface Potential Modeling of Graded-Channel Gate-Stack (GCGS) High-K Dielectric Dual-Material Double-Gate (DMDG) MOSFET and Analog/RF Performance Study ," Silicon, vol. 10, pp. 2865-2875, 2018.

13. S. Dubey, A. Santra, G. Saramekala, M. Kumar, and P. K. Tiwari, "An Analytical Threshold Voltage Model for Triple-Material Cylindrical Gate-All-Around (TMCGAA) MOSFETs," IEEE Transactions on Nanotechnology, vol. 12 , no. 5, pp. 766-774, 2013.

14. R. K. Baruah and R. P. Paily, "A Dual-Material Gate Junctionless Transistor With High- $k$ Spacer for Enhanced Analog Performance," IEEE Transactions on Electron Devices, vol. 61, no. 1, pp. 123-128, Jan. 2014.

15. K. P. Pradhan, S. K. Mohapatra, P. K. Sahu, and D. K. Behera, "Impact of high-k gate dielectric on analog and RF performance of nanoscale DG-MOSFET," Microelectronics Journal, vol. 45, no. 2, pp. 144-151, 2014.

16. Achinta Baidya, Srimanta Baishya, and Trupti Lenka, "Impact of thin high-k dielectrics and gate metals on RF characteristics of 3D double gate junctionless transistor," Materials Science in Semiconductor Processing, vol. 71, pp. 413-420, 2017.

17. H. Ferhati, F. Djeffal, "Graded channel doping junctionless MOSFET: a potential high performance and low power leakage device for nanoelectronic applications, " Journal of Computational Electronics, vol. 17, pp. 129-137, 2018.

18. S. R. Suddapalli and B. R. Nistala, "A center-potentialbased threshold voltage model for a graded-channel dualmaterial double-gate strained-Si MOSFET with interface charges," Journal of Computational Electronics, vol. 18, no. 4, pp. 1173-1181, Dec 2019.

19. D. Ghosh, M. S. Parihar, G. A. Armstrong, and A. Kranti, "High-Performance Junctionless MOSFETs for Ultralow-Power Analog/RF Applications," IEEE Electron Device Letters, vol. 33, no. 10, pp. 1477-1479, 2012.

20. Yongbo Chen, Mohamed Mohamed, Michael Jo, Umberto Ravaioli , and Ruimin Xu "Junctionless MOSFETs with laterally graded-doping channel for analog/RF applications, " Journal of Computational Electronics, vol. 12, no. 4, pp. 757-764, 2013.

21. Shrey Arvind Singh and Shweta Tripathi "Comparative Analysis of Double Gate Junction Less and Gate Stacked Double Gate Junction Less MOSFETs," Semiconductors, vol. 53, no. 13, pp. 1804-1810, 2019.

22. K. Baral, P. K. Singh, S. Kumar, A. Singh, M. Tripathy, S. Chander, and S. Jit, "2-D analytical modeling of drain and gate-leakage currents of cylindrical gate asymmetric halo doped dual material-junctionless accumulation mode MOSFET," AEU - International Journal of Electronics and Communications, vol. 116, p. 153071, 2020.

23. Qi Xiang, Jung-Suk Goo, J. Pan, Bin Yu, S. Ahmed, John Zhang, and Ming-Ren Lin, "Strained silicon NMOS with nickel-silicide metal gate," in 2003 Symposium on VLSI Technology. Digest of Technical Papers (IEEE Cat. No.03CH37407), June 2003, pp. 101-102.

24. J.-S. Lim, S. E. Thompson, and J. G. Fossum, "Comparison of threshold-voltage shifts for uniaxial and biaxial tensile-stressed n-MOSFETs," IEEE Electron Device Letters, vol. 25, no. 11, pp. 731-733, Nov 2004.

25. W. Zhang and J. G. Fossum, "On the threshold voltage of strained-Si-Si1-xGex MOSFETs," IEEE Transactions on Electron Devices, vol. 52, pp. 263 - 268, 032005.

26. M. J. Kumar, V. Venkataraman, and S. Nawal, "A Simple Analytical Threshold Voltage Model of Nanoscale SingleLayer Fully Depleted Strained-Silicon-on-Insulator MOS- 
FETs," IEEE Transactions on Electron Devices, vol. 53, no. 10, pp. 2500-2506, 2006.

27. Sentaurus Device User Guide,, Synopsys, Inc., Mountain View, CA, USA, 2019.

28. Y. Omura, S. Horiguchi, M. Tabe, and K. Kishi, "Quantum-mechanical effects on the threshold voltage of ultrathin-SOI nMOSFETs," IEEE Electron Device Letters, vol. 14, no. 12, pp. 569-571, Dec 1993.

29. S. R. Suddapalli and B. R. Nistala, "The analog/RF performance of a strained-Si graded-channel dual-material double-gate MOSFET with interface charges," Journal of Computational Electronics, 2020. 